\title{
SEQUENCE POLYMORPHISM OF MITOCHONDRIAL DNA HYPERVARIABLE REGIONS I AND II IN MALAY POPULATION OF MALAYSIA
}

\author{
Bhinu Shova Tuladhar*, Nur Haslindawaty Abd Rashid**, Sundarajula Panneerchelvam** and \\ Norazmi Mohd Nor** \\ *National Forensic Science Laboratory,Khumaltar, Lalitpur P.O.Box.4540 Kathmandu, Nepal. \\ ** School of Health Science, Health Campus, University Science Malaysia, 16150 Kubang Kerian, Kelantan, \\ Malaysia.
}

\begin{abstract}
The analysis of the control region of human mitochondrial genome (mtDNA) especially hypervariable regions I (HVI) and II (HVII) segments have been proven to be useful for human identification. For forensic application of mtDNA profiling in Malaysia, a comprehensive database on both HVI and HVII regions are essential. In order to identify polymorphic positions and to determine their frequency in the Malay population, mtDNA HVI and HVII regions of 103 maternally unrelated individuals were amplified ,sequenced and compared with Cambridge reference sequence (CRS). Sequence comparison led to the identification of a total of 446 and 604 location polymorphisms in mtDNA HVI and HVII regions respectively. This polymorphisms defined by 88 haplotypes ( 81 unique) in the HVI and 78 haplotypes (64 unique) in the HVII regions. In combined HVI and HVII defined 101 haplotypes (99 unique) was defined. In the HVII region All the individuals in HVII showed nucleotide transition event from A_G at nucleotide position 073 and 263 and an insertion of cytosine (315.1C) at nucleotide position 315. The genetic diversity and probability of random match in combined HVI and HVII of 103 Malay individuals was found to be 0.9996 and 0.0101 respectively.
\end{abstract}

Keywords: mtDNA; Polymorphism; HVI; HVII; Haplotype.

\section{INTRODUCTION}

Currently, most of the forensic science laboratories used STR DNA profiling of chromosomal DNA as a standard DNA profiling method. However, when dealing with biological samples of human remains and problematic samples (e.g. old stains, decomposed tissue, hair shaft, teeth or bone) STRs DNA profiling has limitations due to sample conditions caused by environmental effects [1]. In this situation, mtDNA sequence analysis is found to be valid alternative and reliable tool for genetic characterization $[2,3,4]$. mtDNA HVI and HVII are found highly variable in the human population [5]. Since the sequence of these hypervariable regions provides a high degree of information for discriminating between unrelated individuals, the forensic identity testing has focused on sequence variation within these two hypervariable regions $[6,7,8]$. In addition, any living relative may provide a reference sample when an individual is not available for direct comparison with a biological sample.
Malaysia consists of a multiracial population with the Malays, Chinese and Indians forming the main ethnic groups. Published database for both HVI and HVII segments of mtDNA on major ethnic Malay population of Malaysia is almost negligible. For forensic application of mtDNA profiling in Malaysia, a comprehensive database on both HVI and HVII regions are essential. Therefore, in this study 103 maternally unrelated Malay individuals were used for the purposes of mtDNA polymorphism study.

\section{MATERIALS AND METHODS}

\section{DNA extraction}

Buccal swabs were collected from 103 maternally unrelated Malays residing in Peninsular Malaysia. Genomic DNA was extracted from buccal swabs using organic extraction method.

Author for Correspondence: Bhinu Shova Tuladhar, National Forensic Science Laboratory, Khumaltar, Kathmandu, Nepal. E-mail:bhinut@yahoo.com. 


\section{Amplification and sequencing of mtDNA}

PCR amplification was performed using established primers for HVI (L15997-H16391) and HVII (L048-H408) [9]. DNA template (50 ng) were amplified in a $20 \mu \mathrm{l}$ reaction volume containing $160 \mu \mathrm{M}$ dNTPs, $2.5 \mathrm{mM} \mathrm{MgCl}$, 1XPCR buffer, 20 pmol primers [5] and 1.5 unit of Taq polymerase. The PCR condition for both HVI and HVII were $95^{\circ} \mathrm{C}$ for 3 minutes, followed by 30 cycles at $95^{\circ} \mathrm{C}$ for $30 \mathrm{sec}, 60^{\circ} \mathrm{C}$ for $30 \mathrm{sec}, 72^{\circ} \mathrm{C}$ for 30 sec, followed by $72^{\circ} \mathrm{C}$ for 5 minutess. Amplified PCR products were purified using QIAquick gel purification kit (QIAGEN, Germany) and was sequenced using ABI 3700 DNA sequencer (PE Applied Biosystems, USA). The sequencing was performed in both directions using the same forward and reverse primers.

\section{Analysis of data}

The nucleotide positions 15997-16391 (HVI) and 048408 (HVII) were analysed [10]. The haplotype diversity (h) was calculated according to Tajima [11] and random match probability (p) was calculated according to Stoneking et al [5].

\section{RESULTS}

The sequence data of all 103 unrelated ethnic Malay individuals were compared with CRS [10]. The analysis of mtDNA HVI of 103 Malay individuals shown 446 location polymorphisms and 88 different haplotypes (81 unique) was observed. In mtDNA of 103 individuals a total of 604 location polymorphisms and 78 different haplotypes (64 unique) was identified. In combined data of HVI and HVII, a total of 101 different haplotypes were recorded with 99 individuals show unique haplotype sequence. The haplotype results and other parameters for mtDNA HVI and HVII of the 103 Malays are presented in Table 1 and Table 2 respectively. The genetic diversity for 103 Malays for HVI and HVII was 0.9958 and 0.9905 respectively. The probability of random match of HVI and HVII among 103 Malays was 0.0139 and 0.0191 respectively. The genetic diversity and probability of random match of in combined HVI and HVII was 0.996 and 0.0101 respectively.

\section{DISCUSSION}

Both mtDNA HVI and HVII regions exhibit transitions as the most frequently occurring events within sequence deviations with $85.87 \%$ and $56.46 \%$ respectively. However, the majority of transition in mtDNA HVI region is pyrimidine transition $35.77 \%(\mathrm{C} \rightarrow \mathrm{T})$ whereas in the mtDNAHVII region, the majority is purine transition $64.51 \%(\mathrm{~A} \rightarrow \mathrm{G})$, This purine $\mathrm{A} \rightarrow \mathrm{G}$ transition in HVII also found occurring more frequently in other populations with in Korean 70.12\% [13], German 62.68\% [14], Southern Spanish 58.4\% [15], Caucasian Danes 58.04\% [16] and Brazilian 97.5\% [17]. The nucleotide position 16223 exhibits the most frequent $\mathrm{C}_{\rightarrow} \mathrm{T}$ transition with a distribution of $37.22 \%$ within Malay population and the same polymorphism also reported in Argentine,
Amerindian, Asian and African populations [12]. In mtDNA HVI, $\mathrm{A}_{\rightarrow} \mathrm{C}$ transversion at nucleotide position 16183 coupled with $\mathrm{T}_{\rightarrow} \mathrm{C}$ transition at nucleotide position 16189 exhibited in 18 Malay individuals. Five individuals exhibited a double transition at nucleotide position 16182 and 16183 coupled with $\mathrm{T}_{\rightarrow} \mathrm{C}$ transition at nucleotide position 16189. This type of C-stretches has also been reported in Italian Caucasians and Solvenians [18]. The insertions in the HVII region was observed at distribution of $35.59 \%$ in Malay individuals whereas in mtDNA HVI region the insertions was observed at lower frequency which is $0.9 \%$. A similar pattern was seen in other populations such as in the Korean 22.62\% [13], German 32.58\% [14], Polish $37.26 \%$ [19], Southern Spanish 38.53\% [15], Caucasian Danes 34.30\% [16] and Brazilian 21.18\% [17]. In mtDNA HVII region all the Malay individuals showed nucleotide transition event from $\mathrm{A}_{\rightarrow} \mathrm{G}$ at nucleotide position 073 and 263 and an insertion of cytosine (315.1C) at nucleotide position 315 . The other insertions were also identified at nucleotide positions at $309(+\mathrm{C}$, $+2 \mathrm{C}$ ) in 98 individuals (78 individuals with $309.1 \mathrm{C}$ and 20 individuals with $309.1 \mathrm{C}, 309.2 \mathrm{C}$ ). The presence of insertion $\mathrm{C}$ residues between nucleotide positions 303 309 and 311-315 in mtDNA HVII region also reported in many populations $[12,13,14,15,16,17,19,20]$.

The most frequent mtDNA HVI haplotype (3.88\%) is defined by polymorphism at nucleotide position 16223 $\mathrm{C} \rightarrow \mathrm{T}$ transiton in Malay population, which also found in South Indians (2.1\%) [9], Argentine population (8.9\%) [12], Taiwanese Han population [21], Japanese population (6\%) [22], Russian (1.59\%) [23], Indigenous Indian tribes (5\%) [24], Korea ethnic Chinese (1.81\%) [25]. The most frequent mtDNA HVII haplotype (6.79 $\%$ ) was defined by polymorphisms at nucleotide positions 073 and $263 \mathrm{~A} \rightarrow \mathrm{G}$ transitions, $249 \mathrm{~A}-\mathrm{del}$ and the insertion of a C-residues at $309.1 \mathrm{C}$ and $315.1 \mathrm{C}$ in Malay population, where the similar polymorphisms also observed in Korea ethnic Chinese (1.81\%) [25], North east Chinese (5.88 \%) [26] and North East Germany population $(0.33 \%)$ [27].

\section{CONCLUSION}

The total number of polymorphic sites in mtDNA HVI (91) and HVII (52) of Malay individuals clearly shows that the control region of mtDNA region is highly polymorphic. The compilation of mtDNA HVI and HVII in this study provides a comprehensive mtDNA database for Malay population of Malaysia that will be useful for forensic purposes.

\section{ACKNOWLEDGEMENT}

Specials thank to Professor Dr. Zainul F. Zainuddin a Former Dean of School of Health Science, USM for his strong support and advice. Appreciation also to Ministry of Science, Technology and the Environment, Malaysia for providing research grants (09-02-05-3122 EA011) 
Table 1: List of mtDNA HVI and HVII haplotypes in the Malay population $(n=103)$.

\begin{tabular}{|c|c|c|}
\hline \multirow{2}{*}{$\begin{array}{l}\text { Sample } \\
\text { No. }\end{array}$} & \multicolumn{2}{|c|}{ mtDNA Haplotype } \\
\hline & HVI (15997-16391) & HVII (048-408) \\
\hline$\overline{M 1}$ & $16108,16129,16162,16172,16240$ & $16304073,195,249 d, 263,309.1 C, 315.1 C$ \\
\hline M2 & $16147,16182.1 C, 16189,16217,16235,16293$ & 073, 249d, 263, 309.1C, 315.1C \\
\hline M3 & $16190,16223,16327,16330$ & $073,113,263,309.1 \mathrm{C}, 309.2 \mathrm{C}, 315.1 \mathrm{C}$ \\
\hline M4 & 16140, 16183C, 16189, 16266A, 16304 & $073,125,128,263,309.1 C, 315 . C$ \\
\hline M5 & 16172,16304 & $073,249 d, 263,309.1 C, 315.1 C$ \\
\hline M6 & $16182 \mathrm{C}, 16183 \mathrm{C}, 16189,16223$ & $073,088,263,309.1 \mathrm{C}, 315.1 \mathrm{C}$ \\
\hline M7 & $16086,16148,16223,16259,16278,16319$ & $073,150,152,200,263,315.1 \mathrm{C}$ \\
\hline M8 & $16091,16223,16298,16327$ & $073,249 d, 263,309.1 C, 315.1 C$ \\
\hline M9 & 16218,16362 & $073,146,150,199,263,309.1 C, 315.1 C$ \\
\hline M10 & 16182C, 16183C, 16189, 16219C & $073,146,263,309.1 \mathrm{C}, 309.2 \mathrm{C}, 315.1 \mathrm{C}$ \\
\hline M11 & $16223,16295,16362$ & $073,146,199,263,309.1 \mathrm{C}, 315.1 \mathrm{C}$ \\
\hline M12 & $16093,16209,16223,16263,16278,16319$ & $073,146,150,151,263309.1 C, 309.2 C, 315.1 C$ \\
\hline M13 & $16140,16189,16355$ & $073,146,150,151,263,309.1 \mathrm{C}, 309.2 \mathrm{C}$ \\
\hline M14 & 16223 & 073, 150, 195A, 263 \\
\hline M15 & $16126,16184 A, 16223,16278$ & $073,263,315.1 \mathrm{C}$ \\
\hline M16 & $16172,16183 C, 16189,16223$ & $073,185,189,263,309.1 \mathrm{C}, 315.1 \mathrm{C}$ \\
\hline M17 & $16176,16278,16354$ & $073,199,263,315.1 \mathrm{C}$ \\
\hline M18 & $16027 \mathrm{G}, 16074.1 \mathrm{C}, 16140,16183,16189,16266 \mathrm{~A}$ & $073,210,263,309.1 \mathrm{C}, 315.1 \mathrm{C}$ \\
\hline M19 & 16037, 16192, 16288, 16304, 16309, 16390 & $073,143,183,263,309.1 \mathrm{C}, 309.2 \mathrm{C}, 315.1 \mathrm{C}$ \\
\hline M20 & $16037,16129,16148,16183 C, 16189,16319$ & $073,093,113,263,315.1 \mathrm{C}$ \\
\hline M21 & 16140,16189 & $073,210,263,309.1 \mathrm{C} 315.1 \mathrm{C}$ \\
\hline M22 & $16108,16129,16162,16172,16304$ & $073,150,249 d, 263,309.1 C, 315.1 C$ \\
\hline M23 & $16126,16181,16209,16362$ & $073,152,242,263,309.1 \mathrm{C}, 315.1 \mathrm{C}$ \\
\hline M24 & 16223 & $073,146,150,199,263,309.1 C, 315.1 C$ \\
\hline M25 & $16126,16231,16311,16362$ & $073,204,207,263,309.1 C, 309.2 C, 315.1 C$ \\
\hline M26 & $16223,16295,16362$ & $073,146,199,263,315.1 \mathrm{C}$ \\
\hline M27 & $16129,16140,16271,16364,16367 G$ & $073,143,146,151,263,315.1 \mathrm{C}$ \\
\hline M28 & $16177,16223,16263,16266,16274,16311,16343 \mathrm{G}$ & 073, 146, 152, 263 309.1C, 315.1C \\
\hline M29 & $16129,16172,16304$ & $073,249 d, 263,309.1 C, 309.2 C, 315.1 C, 332,362$ 366, 389, 394A \\
\hline M30 & $16108,16129,16162,16172,16278,16304$ & $073,249 d, 263,309.1 C, 315.1 C, 366$ \\
\hline M31 & $16192,16223,16304,16309,16390$ & $073,263,309.1 \mathrm{C}, 315.1 \mathrm{C}$ \\
\hline M32 & $16093,16223,16243,16270,16319,16352$ & $073,121 \mathrm{~T}, 195,204,227,263,309.1 \mathrm{C}, 315.1 \mathrm{C}$ \\
\hline M33 & $16188,16209,16223,16325$ & $073,263,309.1 \mathrm{C}, 315.1 \mathrm{C}$ \\
\hline M34 & $16162,16172,16304,16366 \mathrm{G}$ & $073,249,263,309.1 \mathrm{C}, 309.2 \mathrm{C}, 315.1 \mathrm{C}$ \\
\hline M35 & $16136,16261,16292,16294$ & 073, 086.1G, 207, $263309.1 \mathrm{C}, 315.1 \mathrm{C}$ \\
\hline M36 & $16223,16295,16362$ & 073, 085d, 094.1G, 199, 263, 309.1C \\
\hline M37 & $16145,16181,16192,16223,16291,16304$ & $073,210,263,309.1 \mathrm{C}, 315.1 \mathrm{C}$ \\
\hline M38 & $16172,16223,16261$ & $073,097,200,263,309.1 C, 315.1 C$ \\
\hline M39 & $16027 G, 16260,16298,16355,16362,16367,16383$ & $073,207,249 d, 263,309.1 \mathrm{C}, 315.1 \mathrm{C}$ \\
\hline M40 & 16108 A, 16129, 16162, 16172 & $073,249 d, 263,309.1 C, 315.1 C$ \\
\hline M41 & $\begin{array}{l}16037 d, 16140,16182 C, 16183 C, 16189,16217,16278, \\
16335,16343 C\end{array}$ & $073,152,263,309.1 \mathrm{C}, 315.1 \mathrm{C}, 366$ \\
\hline M42 & $\begin{array}{l}\text { 16037, } 16140,16183 C, 16189,16213,16217,16242 \\
16274,16335\end{array}$ & $073,085 d, 146,150,195,263,309.1 C, 315.1 C$ \\
\hline M43 & 16037, 16140, 16183C, 16189, 16261, 16267A, 16274 & $073,085 d, 152,210,263,309.1 C, 315.1 C$ \\
\hline M44 & $16223,16261,16362,16390$ & $073,152,263,309.1 \mathrm{C}, 315.1 \mathrm{C}$ \\
\hline M45 & $16223,16257 A, 16261,16292,16294$ & $073,085,150,263,309.1 \mathrm{C}, 315.1 \mathrm{C}$ \\
\hline M46 & $16126,16184 \mathrm{~A}, 16223,16278$ & $073,263,315.1 \mathrm{C}$ \\
\hline M47 & $16091,16223,16298,16327$ & 073, 94.1G, 151.1 G, 249d, 263, 315.1C \\
\hline M48 & $16108,16129,16162,16172,16278,16304$ & 073, 085d, 249 d, 263, 309.1C, 315.1C \\
\hline M49 & $16111,16223,16278,16291$ & $073,204,207,263,309.1 C, 309.2 C, 315.1 C, 321,332$ \\
\hline M50 & $16108,16129,16162,16172,16304$ & $073,249 d, 263,315.1 C$ \\
\hline M51 & $16126,16231,16311$ & 073, 085d, 094.1G, 204, 207, 263, 309.1C, 309.2C, 315.1C \\
\hline M52 & $16140,16183 C, 16189,16362$ & 073, 085d, 092.1T, 094.1G, 097, 210, 263, 309.1C, 309.2C, 315.1C \\
\hline M53 & 16223 & 073, 094.1G, 124, 150, 195A, 263, 315.1C \\
\hline
\end{tabular}

Scientific World, Vol. 12, No. 12, September 2014 


\begin{tabular}{|c|c|c|}
\hline M54 & 16223 & $073,146,150,199,263,309.1 C, 315.1 C$ \\
\hline M55 & $16140,16182 \mathrm{C}, 16183 \mathrm{C}, 16217,16274,16335$ & $073,085 d, 146,195,263,309.1 C, 309.2 C$ \\
\hline M56 & 16182C, $16183 \mathrm{C}, 16189,16266 \mathrm{~A}$ & 073, 146, 263, 309.1C, 315.1C, 389 \\
\hline M57 & $16129,16188,16209,16223,16325$ & $073,085 d, 263,309.1 C, 315.1 C$ \\
\hline M58 & 1603d, 16136, 16223, 16257A, 1626116294 & 073, 094.1G, 207, 263, 309.1C, 315.1C \\
\hline M59 & $16027 \mathrm{G}, 16129,16192,16223,16258 \mathrm{C}, 16289,16297$ & $073,150,263,309.1 \mathrm{C}, 315.1 \mathrm{C}, 332$ \\
\hline M60 & $16037,16140,16183 C, 16190$ & 073, 151.1G, 210, 263, 309.1C, 315.1C \\
\hline M61 & $16295,16362,16380$ & $07,146,199,263,309.1 \mathrm{C}, 309.2 \mathrm{C}, 315.1 \mathrm{C}$ \\
\hline M62 & $16192,16304,16309,16390$ & 073, 094.1G, 124, 263, 309.1C, 315.1C \\
\hline M63 & $16140,16183 \mathrm{C}, 16189,16274,16372 \mathrm{G}$ & $073,085 d, 210,263,315.1 C$ \\
\hline M64 & $16223,16311,16362$ & $073,146,152,187,263,315.1 \mathrm{C}$ \\
\hline M65 & $16129,16172,16304$ & 073, 094.1G, 124, 249d, 263, 309.1C, 309.2C, 315.1C \\
\hline M66 & 16027G, 16108, 16129,16172, 16304, 16364G, 16367 & $073,149,263,309.1 \mathrm{C}, 315.1 \mathrm{C}$ \\
\hline M67 & $16037,16129,16190,16223,16234,16290$ & 073, 094.1G, 146, 195, 263, 315.1C \\
\hline M68 & 16196T,16223, 16233C, 16273Т, 16291 & $073,263,309.1 C, 315.1 C$ \\
\hline M69 & $16223,16261,16362,16367,16382 \mathrm{G}, 16390$ & $073,146,150,195,263,315.1 \mathrm{C}$ \\
\hline M70 & 16027G, 16074.1C, 16129, 16172, 16304, 16367,16382 & $073,150,249 d, 263,315.1 C$ \\
\hline M71 & $16183 C, 16189,16223,16372$ & $073,124,263,309.1 C, 315.1 C$ \\
\hline M72 & $16177,16263,16266,16274,16311,16343$ & $073,146,152,263,309.1 \mathrm{C}, 315.1 \mathrm{C}$ \\
\hline M73 & 16295,16362 & $073,146,199,263,309.1 \mathrm{C}$ \\
\hline M74 & $16223,16261,16362,16390$ & $073,146,187,263,315.1 \mathrm{C}$ \\
\hline M75 & $16209,16223,16233 C 16274$ & $073,143,146,263,309.1 \mathrm{C}, 315.1 \mathrm{C}$ \\
\hline M76 & 16183,16223 & $073,113,263,309.1 \mathrm{C}, 309.2 \mathrm{C}, 315.1 \mathrm{C}, 375$ \\
\hline M77 & $16129,16189,16223,1633$ & $073,121 \mathrm{~T}, 263,315.1 \mathrm{C}$ \\
\hline M78 & 16196T, 16223, 16291, 16362 & $073,263,309.1,315.1 \mathrm{C}$ \\
\hline M79 & $16108,16129,16162,16172,16304$ & 073, 094.1G, 249d, 263, 315.1C \\
\hline M80 & $16223,16325,16362$ & $073,199,263,309.1 \mathrm{C}, 309.2 \mathrm{C}, 315.1 \mathrm{C}$ \\
\hline M81 & $\begin{array}{l}\text { 16051, 16156, 16219C, 16223,16233C, 16258, } 16362 \\
16384,16390\end{array}$ & $073,195,263,309.1 \mathrm{C}, 315.1 \mathrm{C}$ \\
\hline \multicolumn{2}{|c|}{ M82 16079, 16171, 16209, 16243, 16278} & $073,195,263,309.1 C, 315.1 \mathrm{C}$ \\
\hline M83 & $16108,16129,16162,16172,16304$ & $073,249 d, 263,309.1 C, 315.1 C$ \\
\hline M84 & $16129,16272,16311$ & 073, 199, 249d, 263, 309.1C, 309.2C, 315.1C, 316 \\
\hline M85 & 16223,16311 & $073,146,263,309.1 \mathrm{C}, 315.1 \mathrm{C}$ \\
\hline M86 & $16223,16261,16294$ & $073,152,263,315.1 \mathrm{C}$ \\
\hline M87 & $16129,16172,16304$ & $073,249 d, 263,309.1 C, 315.1 C$ \\
\hline M88 & $16093,16129,1622316261,16356$ & $073,150,152,263,309.1 \mathrm{C}, 315.1 \mathrm{C}$ \\
\hline M89 & $16147,16182.1 \mathrm{C}, 16189,16217,16235$ & $073,146,263,309.1 \mathrm{C}, 309.2 \mathrm{C}, 315.1 \mathrm{C}$ \\
\hline M90 & $16176,16223,16278,16354$ & $073,199,263,315.1 \mathrm{C}$ \\
\hline M91 & $16129,16172,16256,16305,16309$ & $73,152,263,309.1 \mathrm{C}, 315.1 \mathrm{C}$ \\
\hline M92 & $16288,16304,16309,16390$ & $073,085 \mathrm{~d}, 143,183,263,309.1 \mathrm{C}, 309.2 \mathrm{C}, 315.1 \mathrm{C}$ \\
\hline M93 & $16129,16223,16259,16278,16291,16362$ & $073,195,263,286,292,293,294,309.1 C, 315.1 C$ \\
\hline M94 & 16037d, 16140, 16183C, 16189, 16266A, 16298 & 073, 092T, 097,210, 263, 309.1C, 315.1C, 366 \\
\hline M95 & $16037,16140,16183 C, 16189$ & $073,210,263,309.1 \mathrm{C}, 315.1 \mathrm{C}$ \\
\hline M96 & $16134,16223,16362$ & $073,263,309.1 \mathrm{C}, 315.1 \mathrm{C}, 366,383$ \\
\hline M97 & $16093,16223,16295,16362$ & $073,150,199,263,309.1 \mathrm{C}, 309.1 \mathrm{C}, 315.1 \mathrm{C}, 366,380,389$ \\
\hline M98 & $16223,1623416300,16311$ & $073,146,263,315.1 \mathrm{C}$ \\
\hline M99 & $16126,16129,16192,16223,16297$ & $073,146,199,263,309.1 \mathrm{C}, 315.1 \mathrm{C}$ \\
\hline M100 & $16147,16189,16217,16235$ & $073,263,309.1 \mathrm{C}, 315.1 \mathrm{C}$ \\
\hline M101 & $16086,16156,16189,16223,16234$ & $073,210,263,315.1 \mathrm{C}$ \\
\hline M102 & $16209,16298,16355,16362$ & $073,152,207,249 d, 263,309.1 C, 315.1 C$ \\
\hline M103 & $16223,16257 \mathrm{~A}, 16261,16292,16294$ & $073,150,207,263,309.1 \mathrm{C}, 309.2 \mathrm{C}, 315.1 \mathrm{C}$ \\
\hline
\end{tabular}


Table 2: Distribution of location polymorphisms of mtDNA HVI and HVII in Malay population $(n=103)$.

\begin{tabular}{lllll}
\hline Mutation Type & \multicolumn{2}{c}{ HVI } & \multicolumn{2}{c}{ HVII } \\
\hline & $\begin{array}{l}\text { Number } \\
\text { of } \\
\text { positions }\end{array}$ & $\begin{array}{l}\text { Total number } \\
\text { of } \\
\text { mutations }\end{array}$ & $\begin{array}{l}\text { Number } \\
\text { of } \\
\text { positions }\end{array}$ & $\begin{array}{l}\text { Total } \\
\text { number of } \\
\text { mutations }\end{array}$ \\
\hline Transitions & & & & \\
\hline C-T & 26 & 137 & 7 & 29 \\
T-C & 22 & 153 & 7 & 63 \\
A-G & 16 & 46 & 6 & 220 \\
G-A & 7 & 47 & 9 & 29 \\
\hline Total & $\mathbf{7 1}$ & $\mathbf{3 8 3}$ & $\mathbf{2 9}$ & $\mathbf{3 4 1}$
\end{tabular}

\begin{tabular}{lllll}
\hline Transversions & & & & \\
C-A & 5 & 11 & 2 & 2 \\
A-C & 5 & 29 & 0 & 0 \\
C-G & 3 & 5 & 0 & 0 \\
G-C & 0 & 0 & 1 & 1 \\
T-G & 2 & 7 & 2 & 2 \\
G-T & 2 & 3 & 3 & 5 \\
T-A & 0 & 0 & 1 & 2 \\
\hline Total & $\mathbf{1 7}$ & $\mathbf{5 5}$ & $\mathbf{9}$ & $\mathbf{1 2}$ \\
\hline
\end{tabular}

\begin{tabular}{lllll}
\hline Insertions & & & & \\
$+\mathrm{C}$ & 2 & 4 & 3 & 201 \\
$+\mathrm{G}$ & 0 & 0 & 3 & 13 \\
$+\mathrm{T}$ & 0 & 0 & 1 & 1 \\
\hline Total & $\mathbf{2}$ & $\mathbf{4}$ & $\mathbf{7}$ & $\mathbf{2 1 5}$ \\
\hline
\end{tabular}

\section{Deletions}

\begin{tabular}{lllll}
$-\mathrm{A}$ & 1 & 4 & 2 & 21 \\
$-\mathrm{G}$ & 0 & 0 & 2 & 12 \\
$-\mathrm{T}$ & 0 & 0 & 3 & 3 \\
\hline Total & $\mathbf{1}$ & $\mathbf{4}$ & $\mathbf{7}$ & $\mathbf{3 6}$ \\
\hline $\begin{array}{l}\text { Total } \\
\text { polymorphic } \\
\text { positions }\end{array}$ & $\mathbf{9 1}$ & $\mathbf{4 4 6}$ & $\mathbf{5 2}$ & $\mathbf{6 0 4}$ \\
\hline
\end{tabular}

\section{REFERENCES}

[1] Schneider, P. M., Bender, K., Mayr, R. W., Parson, W., Hoste, B., Decorte, R., Cordonnier, J., Vanek, D., Morling, N., Karjalainen, M., Carlotti, C. M. P., Sabatier, M., Hohoff, C., Schmitter, H., Pflug, W., Wenzel, R., Patzelt, D., Lessig, R., Dobrowolski, P., O’Donnell, G., Garafano, L., Dobosz, M., Knijff, P. de., Mevag, B., Pawlowski, R., Gusmăo, L., Vide, M. C., Alonso, A. A., Ferma'ndez, O. G., Nicolás, P.S., Kihlgreen, A., Bär, w., Meier, V., Teyssier. A., Coquoz, R., Brandt, C., Germann, U., Gill, P., Hallett, J. and Greennalgh, M. 2004. STR analysis of artificially degredaded DNA-results of a collaborative European exercise. Forensic Sci. Int. 139: 123-134.

[2] Rousselet, F. and Mangin, P. 1998. Mitochondrial DNA polymorphicm: a study of 50 French Caucasian individuals and application to forensic casework. Int J legal Med. 111: 292-298.

[3] Budowle, B., Wilson, M. R., DiZinno, J. A., Stauffer, C., Fasano, M. A, Holland, M. M. and Monson, K. L. 1999. Mitochondrial DNA regions HVI and HVII population data. Forensic Sci. Int. 103: $23-35$.

[4] Seo,Y., Uchiyama, T., Matsuda, H., Shimizu, K., Takami, Y., Nakayama, T. and Takahama, K. 2002. Mitochondrial DNA and STR typing of matter adhering to an earphone. J Forensic Sci. 47 (3): 605-608.

[5] Stoneking, M., Hedgecodk, D., Higuchi, R.G., Vigilant, L. and
Erlich, H.A. 1991. Population variation of human mt.DNA control region sequences detected by enzymatic amplification and sequence specific oligonucleotide probes. Am. J. Hum. Genet. 48: 370-382.

[6] Watson, E., Forster, P., Richards, M. and Bandelt, H. J. 1997. Mitochondrial footprints of human expansion in Africa. Am. J. Hum. Genet. 61: 691-704.

[7] Parson, W., Parsons, T. J., Scheithauer, R. and Holland, M. M. 1998. Population data for 101 Austrian Caucasian mitochondrial DNA d-loop sequences: application of mtDNA sequence analysis to a forensic case. Int J Legal Med. 111 (3): 124-132.

[8] Parson, W., Brandstätter, A., Pircher, M., Steinlechner, M. and Scheithauer, R. 2004. EMPOP-the EDNAP mtDNA population database concept for a new generation, high-quality mtDNA database. International Congress Series. 1261: 106-108.

[9] Rajkumar, R. and Kashyap, V. K. 2003. Haplotype diversity in mitochondrial DNA hypervariable regions I and II in three communities of Southern India. Forensic Sci. Int. 136: 79-82.

[10]Anderson, S., Bankier, A. T., Barrell, B. G., Bruijn, M. H. L., Coulson, A. R., Drouin, J., Eperon, I. C., Nierlich, D. P., Roe, B. A., Sanger, F., Schreier, P. H., Smith, A. J. H., Staden, R. and Young, I. G. 1981. Sequence and organization of the human mitochondrial genome. Nature. 290: 457-464.

[11] Tajima, F. 1989. Statistical method for testing the neutral mutation hypothesis by DNA polymorphism. Genetics.123: 585-595.

[12] Filippini, S.E., Castro, A., Fraga, M.G., Gagliardi, F., Echenique, C. and Di lonardo, M. 2004.Sequence polymorphisms of mitochondrial control region DNA in Argentine population. International Congress Series. 1261: 413-415.

[13] Lee, S.D., Shin, C.H., Kim, K.B., Lee, Y.S. and Lee, J.B. 1997. Sequence variation of mitochondrial DNA control region in Koreans. Forensic Sci. Int. 87: 99-116.

[14] Lutz, S., Weisser, H-J., Heizmann, J. and Pollak, S. 1998. Location and frequency of polymorphic positions in the mtDNA control region of individuals from Germany. Int $J$ Legal Med. 111: 67-77.

[15] López-Soto, M., and Sanz, P. 2000. Mitochondrial DNA variability on D-loop in 46 unrelated individuals living in Andalusia (South of Spain). Progress in Forensic Genetics 8 (Sensabaugh, G.F., Lincoln, P.J. and Olaisen. B. eds). Elsevier Science B.V. The Netherlands.

[16] Santos, M.V., Mendes, C., Carvalho, M., Vide, M.C., Corte-Real, F., and Vieira, D.N. 2004. Mitochondrial variation in the BahiaBrazil population. International Congress Series. 1261: 404-406.

[17] Tagliabracci, A., Turchi, C., Buscemi, L.and Sassaroli, C. 2001. Polymorphism of the mitochondrial DNA control region in Italians. Int. J. Legal Med. 114: 224-228.

[18] Pajnič, I.Z., Balažic, J. and Komel, R. 2004. Sequence polymorphism of the mitochondrial DNA control region in the Slovenian population. Int J Legal Med. 118: 1-4.

[19] Kupiec, T., Branicki, W. and Pawlowski, R. 2000. Polymorphism of the mitochondrial DNA control region in the Polish population. Progress in Forensic Genetics 8 (Sensabaugh, G.F., Lincoln, P.J. and Olaisen. B. eds). Elsevier Science B.V The Netherlands,

[20] Lima, G., Peña, J.A., Sanchez, A., Pontes, M.L., Abrantes, D., Pereira, M.J., Fernández- Fernández, I., Castro, A., Pinheiro, M.F.and Martíne de Pancorbo, M. 2004. Analysis of the HVI and HVII regions of mitochondrial DNA in 100 individuals from North of Portugal. International Congress Series. 1261: 366-368.

[21] Tsai, L.C., Lin, C.Y., Lee, J.C.I., Chang, J.G., Linacre, A. and Goodwin, W. 2001. Sequence polymorphism of mitochondrial 
D-loop DNA in the Taiwanese Han population. Forensic Sci. Int. 119: 239-247.

[22] Koyama, H., Iwasa, M., Maeno, Y., Tsuchimochi, T., Isobe, I., Seko-Nakamura, Y., Monma-Ohtaki, J., Matsumoto, T., Ogawa, S., Sato, B. and Nago, M. 2002. Mitochondrial sequence haplotype in the Japanese population. Forensic Sci. Int. 125: 93-96.

[23] Belyaeva, O., Bermisheva, M., Khrunin, A., Slominsky, P., Bebyakova, N., Khusnutdinova, E., Mikulich, A. and Limborska, S. 2003. Mitochondrial DNA variations in Russian and Belorusian populations. Hum. Biol. 75: 647-660.

[24] Banerjee, J., Trivedi, R. and Kashyap, V.K. 2005. Mitochondrial DNA control region sequence polymorphism in four indigenous tribes of Chotanagpur plateau, India. Forensic Sci. int. 94 (5): 459460 .

[25] Zhang, Y.Ji., Xu,Q.S., Cui, H., Cui, Y., Lin, H.Y., Kim, K. and Lee, J. 2005b. Haplotype diversity in mitochondrial DNA hypervariable region I, II and III in a Korean ethnic group from Northeast China. Forensic Sci. Int. 151: 299-301.

[26] Zhang, Y.Ji., Xu, Q.S., Zheng, Z.J., Lin, H. Y. and Lee, J. B. 2005a. Haplotype diversity in mitochondrial DNA hypervariable region I, II and III in northeast China Han. Forensic Sci. Int. 149: 267-269.

[27] Poetsch, M., Wittig, H., Krause, D. and Lignitz, E. 2003. Mitochondrial diversity of a northeast German population sample. Forensic Sci. Int. 137: 125-132. 